\title{
Artículos
}

\section{El acceso gratuito a la información bibliográfica: Compludoc y otras bases de datos disponibles en internet}

\author{
Por José Antonio Magán Wals
}

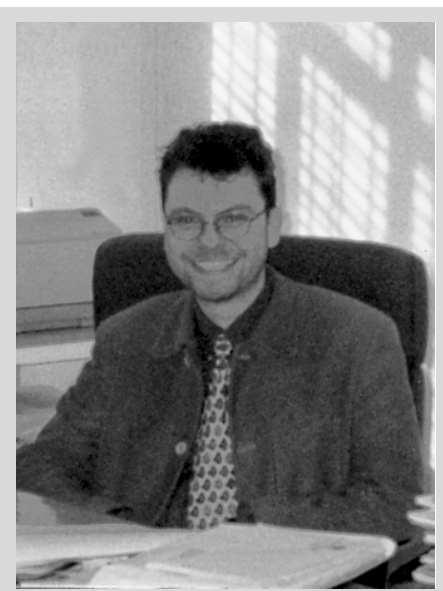

José Antonio Magán Wals

Resumen: Revisión de las principales bases de datos bibliográficas gratuitas en internet sobre libros, revistas y material multimedia con especial referencia a la situación española. Se analiza la tendencia hacia la gratuidad que internet ha marcado en el sector de la información, prestando especial atención a Compludoc (base que recoge las principales revistas científicas españolas): sus orígenes, características y metodología de trabajo.

Palabras clave: Bases de datos bibliográficas, Compludoc, Gratuidad de los servicios, Catálogos.

Title: Free access to bibliographical information: Compludoc and other databases available on the internet

Abstract: Review of the principal free bibliographic databases on the internet covering books, magazines and multimedia, with special reference to the situation in Spain. The article examines the tendency towards free access to databases in the information sector via internet. Special attention is paid to Compludoc (a database that gathers information from the main Spanish scientific magazines): its origins, characteristics and methodology of work.

Keywords: Bibliographic databases, Compludoc, Fees for services, Catalogues.

Magán Wals, José Antonio. "El acceso gratuito a la información bibliográfica: Compludoc y otras bases de datos disponibles en internet". En: El profesional de la información, 2000, julio-agosto, v. 9, n. 7-8, pp. 4-12.

\section{La tendencia a la gratuidad en el sector información, un cambio de paradigma}

El desarrollo de internet ha favorecido la profusión de servicios gratuitos que, pese a las suspicacias iniciales sobre su viabilidad y continuidad, tienden a un crecimiento espectacular frente al declive de los de pago. Más aún, las empresas que los ofrecen son las que obtienen los mayores beneficios: desde navegadores, buscadores, portales, servidores de acceso, servicios de correo y alojamiento de páginas, a versiones gratuitas de productos que funcionan mediante cobro en otros medios de distribución, decayendo así, uno tras otro, los esfuerzos por desarrollar servicios de cobro cuya baza diferencial quería ser la calidad. Algo factible finalmente porque, entre otros factores, los costes de distribución son a cargo del usuario final.

Esta corriente no es nueva en el sector información, ya que una de sus características al dispararse el nivel de difusión es la gratuidad (como el caso de la radio o televisión comerciales), afectando ahora al mundo editorial (cada vez más vinculado al contexto de la comunicación) e inmerso en un cambio de paradigma donde los servicios sin coste implican una profunda adaptación de los métodos de trabajo y obtención de beneficios. Algunas empresas han sabido aclimatarse rápidamente dando un salto cualitativo exclusivamente comprensible si se tiene en cuenta la irrupción de un nuevo factor capaz de multiplicar los niveles de ingresos: la publicidad.

Sólo mediante la conjunción entre este agente y los nuevos sistemas de gestión digitales que incrementan la eficacia (y por tanto los beneficios, revolucionando las bases mismas de la actividad económica) se pueden entender pasos como los de Enciclopaedia Britannica (accesible gratuitamente tras abandonar la distribución impresa y ofreciendo utilidades más desarrolladas que en su formato tradicional), servicios de prensa y periódicos, empresas de todo tipo que, junto a prestaciones de pago, presentan una oferta de información sin coste como estrategia de marketing para captar clientes o, yendo más allá, compañías que generan valores bursátiles colosales sobre servicios y productos gratuitos.

http://www.britannica.com

Lo que parece claro es que los distintos sectores empresariales vinculados al mundo de la información han de adaptarse a un nuevo contexto donde los mecanismos usuales de explotación comercial han dejado 
de ser operativos. Las empresas con capacidad de anticipación que han asumido el cambio están obteniendo unos volúmenes de beneficios altísimos, pero aquellas que ven a la Red sólo como un mecanismo complementario están abocadas a replantear drásticamente sus políticas comerciales. Ahora esta corriente está apareciendo con fuerza en el ámbito bibliográfico, donde el cobro por el acceso mediante pago a bases de datos había sido la norma y la gratuidad la excepción.

\section{Contexto general}

La difusión de internet ha posibilitado la aparición de bases de datos bibliográficas de carácter gratuito que están cuestionando los sistemas tradicionales de consulta basados en el cobro. En la actualidad, muchos servicios comerciales y de entidades públicas ofrecen información bibliográfica libre como medio de captar interés hacia servicios más desarrollados como el texto completo. El dilema ya no se presenta en la esfera de la viabilidad o no del mantenimiento de prestaciones de carácter gratuito, sino sobre el grado de calidad de los servicios de cobro respecto a aquellos, muchos de los cuales son comerciales, siendo curiosa, al menos, la perspectiva de servicios públicos restringidos a clientes directos frente a todo un universo de utilidades gratuitas ofertadas por entidades comerciales.

\section{«Los distintos sectores empre- sariales vinculados al mundo de la información han de adaptarse a un nuevo contexto donde los mecanismos usuales de explo- tación comercial han dejado de ser operativos»}

La tendencia al incremento de la calidad (y número) de los servicios gratuitos es imparable en un contexto donde el nivel de accesibilidad lleva implícito un crecimiento directo de aquella. Cuando se realiza la afirmación anterior, hay que basarse en el hecho de que los de naturaleza gratuita están provocando un cambio en los hábitos de muchos usuarios (profesionales de la información o usuarios finales) que están acostumbrándose a usarlos cada vez más. Junto al aliciente de la gratuidad encuentran otros como interfaces y servicios que mejoran a los de pago "tradicionales". Por ejemplo: Amazon.com desde la perspectiva del usuario general no sólo oferta información bibliográfica al igual que un catálogo de biblioteca, sino que permite la adquisición de material multimedia, descripción más detallada de los registros al incorporar

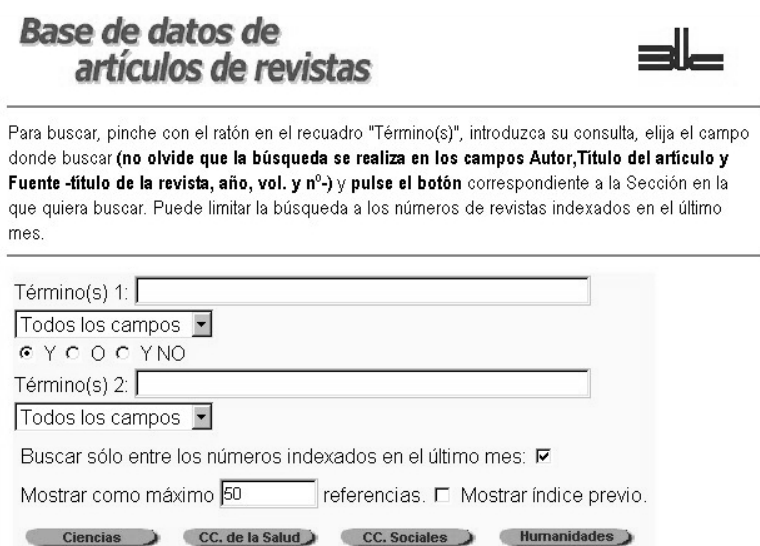

Figura 1. Página de búsquedas de Compludoc

notas sobre su contenido, comentarios de los editores, autores o lectores, foros interactivos, entrevistas a los autores, sugerencias de libros relacionados con el buscado, servicios de alerta en materias o autores de interés, visualización de la cubierta de las obras, sugerencias personalizadas sobre la base de búsquedas anteriores o de usuarios con necesidades similares, etc.

\section{http://www.amazon.com}

Este acaparamiento de accesos y consultas por parte de los nuevos repertorios origina un proceso de retroalimentación, generando un interés progresivo por parte de los editores y autores por ser incluidos. Esta situación ocasiona una mejora directa en la calidad (y cantidad) de sus contenidos. De esta forma Compludoc (que en un primer momento sólo procuraba el sumario de las revistas y un servicio de alerta informativa) comienza a ofrecer, al incrementarse la demanda, un servicio de resúmenes como primer paso hacia otros como el acceso al texto completo para ciertas categorías de usuarios, etc.

\section{http://www.ucm.es/bucm/compludoc}

Por otro lado, la razón esgrimida de la mayor calidad de los servicios de pago parece poco real si se tiene en cuenta que algunas de las más prestigiosas bases de datos bibliográficas se ofrecen de forma gratuita desde hace tiempo en la Red (PubMed, versión gratuita de Medline, Eric, etc.). A ellas se les une un elevado número de otras mucho más específicas, desarrolladas por centros de investigación o por investigadores a título personal y que sólo son accesibles de forma libre, por no argumentar algo tan evidente como que el cobro no siempre implica "calidad".

http://www.ncbi.nlm.nih.gov/PubMed/

\section{http://www.ericae.net/search.htm}

El hecho es que hay una aproximación a un control bibliográfico universal gratuito aunque por vías inesperadas, al tener un papel esencial los propios usuarios y los servicios comerciales - pese a estar fragmentado sectorialmente por instituciones o niveles de actividad, 
y no existir herramientas que permitan búsquedas simultáneas- . Ante ello cabe preguntarse si las bibliotecas no están perdiendo la batalla de la descripción bibliográfica al adoptar una postura mucho más cómoda, como es la adquisición y selección de repertorios bibliográficos en lugar de su creación.

Muchos profesionales argumentarán que los bibliotecarios no son documentalistas (algo muy cuestionable en los tiempos actuales). Pero en un entorno marcado por un cambio radical en los procesos técni$\cos$ - solamente hay que reflexionar sobre la simplificación de la catalogación mediante el uso de herramientas de consulta como Z39.50 o el ofrecimiento, por parte de los distribuidores de libros, de las catalogaciones de los ejemplares como servicio de valor añadido- es lógico pensar que se deben asumir nuevas competencias como agentes productores de información, especialmente de la bibliográfica.

Si bien algunos opinan que la función del bibliotecario ha de orientarse hacia la difusión de la información (cosa obvia por otra parte), parece sensato razonar que ello no implique el abandono en manos de instituciones no bibliotecarias de procesos tan ligados al hacer profesional como la descripción normalizada. Las recientes experiencias respecto a las ventajas de la normalización aconsejan no delegar estas funciones, lo cual no significa que sean las bibliotecas quienes realicen directamente estas tareas, que pueden externalizarse, sino quienes las gestionen controlando su nivel de calidad.

\section{La situación española}

El contexto nacional es especialmente favorable al contar con algunas de las herramientas más avanzadas para un control bibliográfico gratuito. Básicamente, la Agencia Española del Isbn, la Biblioteca Nacional y Compludoc han logrado en los últimos años que el contenido de la información corriente sobre libros y revistas científicas sea accesible sin restricciones. A ello se unen catálogos de bibliotecas que ofertan servicios cada vez más amplios. Así, es posible en estos momentos acceder gratuitamente a un elevado número de recursos bibliográficos que van desde bases de datos comerciales de pago, catálogos más o menos amplios o colectivos (Biblioteca Nacional, Catálogo de las Bibliotecas Públicas del Estado, Catálogo del Cbuc, catálogos de las distintas universidades, etc.) a un número creciente de bases de datos de sumarios de alcance muy variado. Entre estas últimas destaca la excelente Base de dades de sumaris del Cbuc que garantiza un acceso rápido a los sumarios de uno de los más vastos repertorios de revistas extranjeras del estado.

Junto a estos servicios empiezan a aparecer colecciones textuales que permiten augurar un futuro no muy lejano donde el acceso a las recopilaciones digitales suscritas por las bibliotecas de financiación pública será libre. La necesidad de trabajar en esta línea por parte de los profesionales con responsabilidad en la toma de decisiones no debe dejar de apoyarse. Hoy por hoy es muy difícil hacer ver los beneficios de estas políticas que ya han adoptado las empresas comerciales a algunos de nuestros compañeros y autoridades.

\section{«Muchos servicios comerciales y de entidades públicas ofrecen información bibliográfica libre como medio de captar interés hacia servicios más desarrolla- dos como el texto completo»}

Todos estos servicios denotan una concepción bibliotecaria avanzada en consonancia con las más recientes recomendaciones profesionales y políticas, tanto europeas como nacionales. Frente a los temores de principios de los noventa, la realidad de un mundo global ha puesto de manifiesto el valor estratégico de la información, lo cual ha forzado la necesidad de garantizar su acceso indiscriminado como herramienta básica para el desarrollo económico y social. Este incremento de la accesibilidad redunda en claros avances socioeconómicos donde los bibliotecarios tienen mucho que decir, como manifiesta la reciente declaración "de cómo las bibliotecas pueden ayudar a Catalunya a entrar con éxito en la sociedad de la información" realizada por una de las asociaciones profesionales más activas, el $\operatorname{Cobdc}$, en su última reunión y que es todo un decálogo de intenciones al respecto.

\section{Principales servicios gratuitos}

Se pueden clasificar en tres grupos:

1. Bases de datos con información bibliográfica sobre libros. Se incluyen tres tipos de recursos:

- Los catálogos comerciales: existe una oferta editorial amplísima entre la cual destacan sobre todo Amazon.com y Barnes and Noble, quienes han consolidado no sólo un excelente servicio de consulta a información libraria y multimedia sino un volumen de negocio de ventas sorprendente por su rápido nivel de aceptación.

\section{http://www.barnesandnoble.com}

Este tipo de repertorios ha ocasionado la aparición de una nueva relación de los lectores con la información bibliográfica. De hecho, dada la gratuidad de estos catálogos, Bowker's Books in Print, la base de pago en cd-rom de uso profesional, ha sufrido un claro desuso. Por otro lado, frente a los obsoletos catálogos 
(que se limitan a trasladar al nuevo medio la misma información de siempre), las nuevas fuentes comerciales, aunque no se ajustan a la normalización al uso, no sólo ofrecen servicios que los bibliotecarios no ofertamos sino unas interfaces más ágiles, tanto desde el punto de vista estético como de los contenidos y un volumen de referencias que supone en sí un gran salto cuantitativo.
En España se cuenta con una de las más dinámicas, tanto por su volumen de actividad como por la calidad de los servicios que presta, ofreciendo en su base de datos una de las mejoras herramientas para el control gratuito de nuestra producción bibliográfica (un listado de agencias nacionales puede consultarse en la siguiente URL).

http://www.isbn.spk-berlin.de.

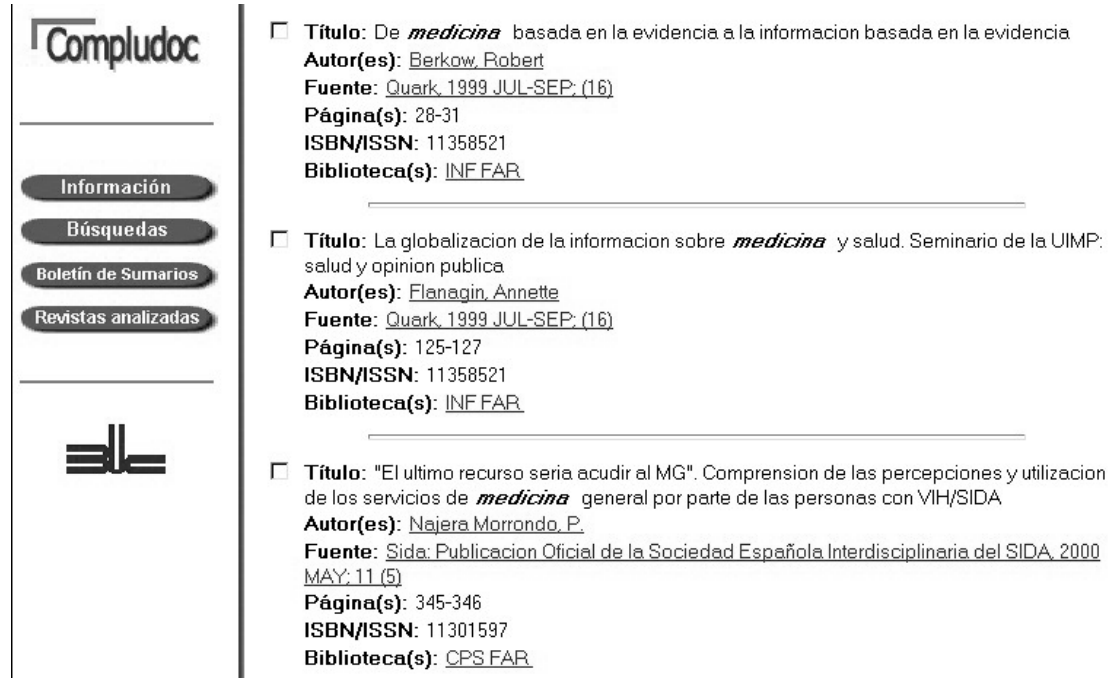

Figura 2. Aspecto de un registro de Compludoc
Junto a la información editorial se ofrece otra (planes de la obra, índices, resúmenes, datos de los editores, comentarios de los autores u otros lectores, etc.), añadiendo un sentido interactivo de la información bibliográfica y un desarrollo visual más atractivo al incorporar la imagen de la cubierta de las obras, publicidad orientada a las necesidades del lector y servicios de alerta de los que adolecen nuestros catálogos. La comparación entre esta información y la ofrecida por las bibliotecas favorecerá la reacción de nuestros profesionales, que aún se resisten a incluir algo tan básico en las descripciones como un resumen del contenido o reseñas relativas a los autores, editores, etc.

Sin embargo, el mercado nacional dista mucho de esta visión pues la propuesta virtual de las librerías es muy restringida, reflejando de forma fiel la crisis que atraviesan y las dudas que mantienen respecto al cambio tecnológico. Aunque la oferta es muy variada, los reducidos volúmenes de venta electrónica inciden en unos desarrollos muy limitados, donde las aventuras comerciales más ambiciosas (Interbook) no terminan de despegar.

\section{http://www.disbumad.es/libreria/index.htm}

- Las bases de datos de agencias bibliográficas nacionales del Isbn.

http://www.mcu.es/bases/spa/isbn/ISBN.html
- Catálogos de bibliotecas. Su oferta es abrumadora, tanto por la calidad de sus servicios como por la extensión de contenidos. Dado que los catálogos comerciales de las librerías y editoriales están dirigidos hacia aquella información que genera mayor volumen de negocio, es en cuanto al libro antiguo y la diversidad cultural e ideológica donde las bibliotecas muestran todo su poder, exhibiendo una cuantiosa oferta no sólo por la pluralidad de contenidos sino también por el alcance de su cobertura cronológica, linguística y geográfica.

El acceso a los catálogos de las grandes bibliotecas, los extensos catálogos colectivos y de entidades científicas y culturales, permite la consulta de materiales en cualquier tipo de lengua sin limitaciones cronológicas o ideológicas. En este ámbito, los frutos de nuestra ética deontológica logran merecidos motivos para sustentar su prestigio plasmándose, gracias a los avances tecnológicos y la filosofía del préstamo interbibliotecario, el carácter universal de la biblioteca en un acceso general a las publicaciones. De este modo, quienes aman la profesión encuentran motivos justificados de orgullo al ver materializarse los esfuerzos de generaciones anteriores de bibliotecarios que han permitido no sólo la salvaguarda de los materiales que hoy día pueden ser consultados, sino la consolidación de una filosofía de servicio que garantiza la difusión gratuita de la información.

Si en un principio con la aparición de internet algunas de las grandes bibliotecas optaron por ofrecer sus catálogos mediante pago, hoy la práctica generalizada es la gratuidad, tendiéndose a ampliar los servicios tradicionales con otros virtuales que configuran la idea, aún difusa, de biblioteca digital. Lógicamente son las grandes bibliotecas nacionales, universitarias, públicas o privadas y los consorcios quienes ofrecen la mejor oferta bibliográfica. Sólo resaltar que las posibilidades de las búsquedas mediante Z39.50 no han hecho más que iniciarse, lo cual facilitará en extremo la consulta de los catálogos y de la información conteni- 
da en ellos, estando por llegar el desembarco de las bibliotecas al mercado de la información, pues si otros ofrecen los puentes, nosotros contamos con los contenidos.

Que esta irrupción permita ampliar la calidad de los servicios públicos depende exclusivamente de nuestra capacidad para concienciar a las autoridades y los profesionales que han de tomar las decisiones relativas a los procesos y productos que ofertar. Tal y como se ha expresado en otros foros, contamos con los medios, y sólo nos falta tener imaginación para organizar los recursos que permitan ofrecer los servicios y contenidos. Con un sistema bibliotecario que opera con miles de profesionales, tenemos la obligación de organizar una cooperación que permita el aprovechamiento de nuestro saber.

\section{Bases de datos con información bibliográfica} de publicaciones periódicas. Abarcan desde los directorios y catálogos de bibliotecas que recogen información sobre las revistas, a las bases de datos que analizan estas publicaciones, entre las cuales destacan las de sumarios de los servicios de acceso al documento.

\section{«Cabe preguntarse si las biblio- tecas no están perdiendo la ba- talla de la descripción bibliográ- fica al adoptar una postura más cómoda, como es la adquisición y selección de repertorios bi- bliográficos en lugar de su cre- ación»}

- Directorios de publicaciones periódicas científicas. El más prestigioso de los gratuitos es el ofrecido por PubList.com que, con información relativa a 150.000 revistas, puede compararse en cobertura y contenidos al Ulrich's international periodical directory (con 157.000 entradas).

http://www.publist.com

- Bases de datos de sumarios. Dentro de las más generales destaca por su volumen UnCover de Carl, que ofrece gratuitamente sumarios de unas 18.000 revistas científicas, siendo la llave de entrada a un servicio de obtención de documentos comercial. Otros ejemplos de recursos gratuitos son NorthernLight (indexa más de 12 millones de artículos de 5.600 publicaciones, noticias de prensa, monografías y 190 millones de páginas web) o Powerize.com, que permite buscar en artículos de 6.000 revistas y 300 periódicos.

http://uncweb.carl.org

http://www.nlsearch.com http://www.infomarket.k-link.com

El más cercano a nosotros es la Base de dades de sumaris ofrecida por el Consorci catalán y que, con más de 6.700 revistas analizadas, constituye una herramienta bibliográfica de primer orden. A ella se añaden otras muchas que están apareciendo, sobre todo a partir de la propuesta comercial de la distribuidora de revistas Ebsco de ofrecer en formato electrónico los datos de los sumarios de las revistas suministradas para uso libre por parte de sus clientes quienes, a partir de ellos, ofrecen bases más o menos extensas según su nivel de contratación. Esta política terminará, previsiblemente, cambiando las reservas de otros distribuidores que ofrecen servicios similares pero con limitaciones respecto a la distribución final por parte de los clientes, puesto que están muy alejadas de las tendencias del mercado y no hacen sino perjudicar a las empresas que las sustentan al no poder proporcionar sus compradores los servicios de quienes trabajan con la competencia.

\section{http://sumaris.cbuc.es/}

Dentro del caso español se puede señalar un elevado número de este tipo de bases de datos, especialmente en la Universidad Politécnica de Cataluña con más de 170.000 registros de 600 revistas o la Uned. Cabe destacar Compludoc, que será analizada posteriormente al presentar características claramente diferenciadoras del resto: a su amplitud de cobertura (más de mil revistas españolas de todas las áreas científicas) añade el hecho de ser desarrollada por personal de la propia biblioteca de la Universidad Complutense, frente a otros servicios que contratan los datos.

\section{http://escher.upc.es/sumaris/indice.htm}

\section{http://193.146.240.166/ConsultaDeSumariosDeRevist} as.html

Por lo tanto, son muchos los recursos disponibles y variadas las formas para su desarrollo (creación propia, a partir de datos proporcionados por los distribuidores de revistas, desde bases de datos suscritas, o mediante la integración gracias a los protocolos actuales de bases externas en catálogos propios, etc.), mostrando una tendencia hacia bases cuyos registros provengan de diferentes sistemas de carga de datos según los intereses, recursos y necesidades estimados por los gestores.

- Bases de datos de resúmenes y especializadas. También existe un elevado número de fuentes de información bibliográficas especializadas en áreas científicas concretas que ofrecen un análisis documental más amplio, incluyendo descriptores o resúmenes en las referencias. Es el caso de PubMed, la versión pública de Medline realizada por la National Library of 


\begin{tabular}{|l|l|l|l|}
\hline \multicolumn{1}{|c|}{ Año } & \multicolumn{1}{|c|}{$\begin{array}{c}\mathbf{N}^{\mathbf{0}} \text { de } \\
\text { referencias /soc }\end{array}$} & $\begin{array}{c}\mathbf{N}^{0} \text { de referencias Compludoc } \\
\text { (sección deciencias sociales) }\left(^{*}\right)\end{array}$ & $\begin{array}{c}\mathbf{N}^{\circ} \text { de referenciasCompludoc(secciónde } \\
\text { humanidades)(*) }\end{array}$ \\
\hline 2000 (hasta abril) & 1.054 & 2.602 & 616 \\
\hline 1999 & 12.608 & 16.540 & 7.381 \\
\hline 1998 & 17.333 & 18.662 & 8.920 \\
\hline
\end{tabular}

\begin{tabular}{|l|l|l|}
\hline \multicolumn{1}{|c|}{ Año } & \multicolumn{1}{|c|}{$\mathbf{N}^{\mathbf{0}}$ de referencias IME } & $\mathbf{N}^{\mathbf{0}}$ de referencias Compludoc(secciónde ciencias de la salud) \\
\hline 2000 (hasta abril) & 0 & 1.798 \\
\hline 1999 & 1.383 & 8.322 \\
\hline 1998 & 3.880 & 8.983 \\
\hline
\end{tabular}

\begin{tabular}{|l|l|l|}
\hline \multicolumn{1}{|c|}{ Año } & \multicolumn{1}{|c|}{$\mathbf{N}^{\mathbf{0}}$ de referencias Icyt } & \multicolumn{1}{|c|}{$\mathbf{N}^{\mathbf{0}}$ de referencias Compludoc(secciónde ciencias)( $\left.{ }^{*} * *\right)$} \\
\hline 2000 (hasta abril) & 200 & 306 \\
\hline 1999 & 5.629 & 2.416 \\
\hline 1998 & 7.109 & 2.320 \\
\hline
\end{tabular}

Figura 3. Tablas

(*) La cobertura temática de Isoc es realizada por dos secciones de Compludoc (que duplican artículos al compartir algunas revistas). $\left(^{* *}\right)$ Hay que tener en cuenta que la UCM carece de estudios de carreras técnicas.

Medicine; Eric, la prestigiosa base de datos de educación, que cuenta con numerosos "espejos" en la Red; Library Reference Center de Ebsco con 150.000 resúmenes de interés para profesionales de la información, o el subset con 80.000 registros de la Civil Engineering Database.

http://www.epnet.com/lrc.html

http://www.pubs.asce.org/cedbsrch.html

En España destacan el excelente Decomate de la Universidad Autónoma de Barcelona que analiza 343 revistas generales; dentro del apartado de bases de datos especializadas, la Universidad Complutense con Psyké, que examina las principales publicaciones de sicología o Enfispo centrada en enfermería, fisioterapia y podología, y Datathéke, sobre biblioteconomía y documentación de la Universidad de Salamanca. Se puede avanzar la inmediata puesta en funcionamiento de EconHis por parte de la biblioteca de la Facultad de ciencias económicas de la Complutense, que cubrirá la información publicada por las principales revistas españolas de economía desde su origen a nuestros días, paso esencial para el control bibliográfico de los estudios económicos en España.

http://decomate.uab.es/

http://cedro.sim.ucm.es/isishtm/psyke.htm

http://cedro.sim.ucm.es/isishtm/enfispo.htm

http://milano.usal.es/dtt.htm\#inicio

http://www.ucm.es/bucm/cee/econhis

3. Bases de datos con información relativa a material audiovisual. Es en este ámbito donde los recursos gratuitos presentan una oferta más amplia y completa que las comerciales. De hecho, una de las grandes carencias del control bibliográfico era en relación con este tipo de soportes. Así, han surgido un ele- vado número de bases de datos, como los metabuscadores de obras y estilos musicales (Argus Music Searcher, Library of Musical Links, etc.), archivos de películas y documentales (Internet Movie Database, All Movie Guide, etc.), así como buscadores de ficheros multimedia, entre los que destaca Richmedia de Lycos.

http://home.sn.no/-kbreivik/argus

http://www.wco.com/jrush/music

http://www.imdb.com

http://www.allmovie.com

http://richmedia.lycos.com

Aunque es criticable su inclusión en este apartado, se engloban también los buscadores y portales de recursos en internet. Dado que son bien conocidos sólo se hará referencia a aquellos que han supuesto una mayor innovación por sus interfaces de consulta y motores de búsqueda: Altavista, Lycos, o Yahoo, primero en incorporar bibliotecarios para la organización de la información, criterio seguido por otros, como Internet Public Library de la University of Michigan o el proyecto de la University of Wisconsin-Madison Scout Report Signpost que selecciona 8.000 recursos gratuitos de calidad. Más especializados, y con el mismo principio, se encuentran en España Guíame! de Esade o EconRed de la Facultad de económicas de la UCM.

http://www.altavista.com

http://www.lycos.es

http://www.yahoo.es

http://www.ipl.org/ref

http://www.signpost.org/signpost

http://www.guiame.net/

http://www.ucm.es/bucm/ceeleconred.htm 


\section{El caso de Compludoc, un servicio académico de información bibliográfica}

Dentro de esta tendencia de creación de bases de datos bibliográficas gratuitas, destaca la puesta en funcionamiento hace tres años por parte de la biblioteca de la Universidad Complutense de Madrid de Compludoc, base de datos que analiza gran parte de las principales revistas científicas españolas recibidas por esta universidad (y algunas de las más prestigiosas extranjeras con mayor impacto).

\section{«Frente a los obsoletos catálo- gos, las nuevas fuentes comer- ciales no sólo ofrecen servicios que los bibliotecarios no oferta- mos sino unas interfaces más ágiles y un volumen de referen- cias que supone en sí un gran salto cuantitativo»}

1. Qué es. Con más de 130.000 accesos, 38.000 búsquedas atendidas y 1.200 DSIs suministrados gratuitamente vía correo electrónico en el mes anterior a la confección de este artículo, es un instrumento primordial sobre la información aparecida en las principales publicaciones científicas españolas, labor posible gracias a las funciones que el personal auxiliar y paraprofesional (becarios, alumnos en prácticas y objetores del servicio militar) ha asumido en los últimos años.

Contiene descripciones desde 1994 y en la actualidad cuenta con más de 160.000 registros pertenecientes a más de mil publicaciones científicas españolas, con un incremento mensual que supera los 5.000 registros. Ha sido desarrollada exclusivamente por la Universidad Complutense mediante la colaboración de los Servicios informáticos, alumnos en prácticas de la Escuela de biblioteconomía y documentación (sólo en su fase de puesta en marcha), el personal de la biblioteca y el apoyo de las autoridades académicas.

Pese a las dudas iniciales sobre la viabilidad del proyecto ante la falta de experiencias similares realizadas por bibliotecas, sus actuales cuotas de utilización parecen señalar el acierto de la apuesta, siendo el servicio más usado del web de la biblioteca complutense, lo cual está motivando que algunos centros participantes abandonen la intención inicial de incluir sólo los sumarios de las revistas para realizar una descripción bibliográfica más exhaustiva y, de forma restringida a la red complutense, se han empezado a ofrecer otras 2.000 revistas extranjeras suscritas.
La base de datos se estructura en dos grandes apartados: uno de búsquedas y otro que permite navegar hasta los registros mediante un sistema hipertextual desde las revistas, sus sumarios, los centros que las poseen o los autores. Admite la realización de servicios como el de DSI vía correo electrónico, la generación de boletines de sumarios electrónicos por centros o la petición automática de envío de los documentos vía préstamo interbibliotecario.

$\mathrm{Su}$ interfaz fue creada mediante herramientas gratuitas accesibles en internet, estando adaptada a las necesidades específicas de nuestros usuarios, pues en el momento de su desarrollo ninguno de los programas de gestión bibliotecaria o documental permitían realizar algunas de las funciones que Compludoc ofrece. $\mathrm{Al}$ estar desarrollado bajo Wais, permite su integración con formularios de consultas Z39.50, por lo que tolera su implementación con otros recursos de información como catálogos, bases de datos, tesauros y revistas digitales.

El proyecto surgió en la biblioteca de la Facultad de ciencias económicas y empresariales bajo el nombre de Econdoc, uniéndose de forma inmediata las de Ciencias políticas y sociología (con la creación de Cpsdoc) y Ciencias de la información (Infdoc), contando con el asesoramiento de la Biblioteca Europea y el Servicio informático de Somosaguas que desarrolló la interfaz de consulta y el programa para la introducción e indexación de los datos. A partir de los resultados iniciales, el equipo de dirección de la biblioteca asumió la expansión del proyecto al resto de los centros, encargando su desarrollo y coordinación a la Unidad de tecnología y sistemas en colaboración con los Servicios informáticos, siendo actualmente mantenida por personal auxiliar y becarios-colaboradores.

2. Características. Su principal objetivo era la rapidez en la actualización a fin de evitar retrasos en el control del contenido de las revistas españolas, pues mientras se tenía información bibliográfica de la literatura extranjera, se carecía de información al día sobre la producción nacional.

\section{«Puesto que el objetivo de Com- pludoc es ofrecer de forma rápi- da información somera, la op- ción de inserción o no de resú- menes depende de los recursos de los centros implicados»}

La descripción empleada aporta información relativa al autor, título, fuente, colación, paginación y, de forma optativa, un resumen indicativo y descriptores. Se dejó de lado la uniformidad en los niveles de des- 
cripción y el control exhaustivo de autoridades en aras de una mayor prontitud en la disposición al público de la información. A estos aspectos técnicos, se sumaban otros conceptuales: el sistema debía ser simple, capaz de ser mantenido por personal del área auxiliar (pues se pretendía sustituir el tedioso trabajo de creación de los boletines de sumarios fotocopiados por un producto más acorde con las nuevas tecnologías), incluir aquellos títulos más demandados por nuestros usuarios y garantizar la consulta gratuita a fin de potenciar su difusión. De hecho, el número de búsquedas de datos restringidos a la $U C M$ es veinte veces menor que al apartado sin restricciones.

Es utilizada casi en un $90 \%$ desde fuera de la red de la Universidad Complutense, siendo la mitad de sus accesos nacionales y un $10 \%$ de países latinoamericanos lo que denota un interés general por la base y no sólo local, pese a que su cobertura se restrinja a revistas suscritas por la $U C M$. Los horarios de utilización se encuentran muy repartidos, aunque es durante las horas intermedias del día y la noche cuando se realiza el mayor número.

\section{Desarrollo y metodología.}

a. Fase de desarrollo de la interfaz Sideria y del programa de generación de la base de datos. El Servicio informático de Somosaguas de la Universidad utilizó para ello el indexador Freewais, sustituido después por Isite del Cnidr, todo ello basado en Wais. Posteriormente, diversos programas desarrollados en Perl fueron configurando la estructura de la base.

b. Fase de introducción de datos. Se ha realizado el proceso de modo diverso: desde listados en ascii puro, al actual sistema basado en una aplicación vía intranet, pasando por otra desarrollada en Visual basic por la biblioteca de económicas. En la actualidad, los centros introducen los datos mediante un sistema del Servicio informático basado en formularios en html dinámico, para posteriormente procesarlos mediante un programa en Perl, preparándolos para su indexación y la generación de las diferentes páginas que permiten navegar a través de los sumarios.

La metodología de trabajo en una primera fase (la de creación de la base retrospectiva) contó para la introducción de datos con alumnos en prácticas de la $E s$ cuela de biblioteconomía y documentación de la UCM coordinados por personal del área auxiliar o técnica. En el siguiente paso, de mantenimiento, el personal auxiliar o paraprofesional era el encargado de la introducción de los datos. La tercera fase, de inclusión de resúmenes informativos (de autor o limitados a la descripción somera de los puntos más destacados dentro del artículo), se realiza tanto por becarios especializados en su área de conocimiento como por el personal de los centros. Puesto que el objetivo de Compludoc es ofrecer de forma rápida información somera, esta opción de inserción o no de resúmenes depende de los recursos de los centros implicados: una vez garantizado el objetivo básico (la recogida del sumario de la revista) son éstos quienes deciden si pueden ampliar el análisis documental.

4. Resultados. Dadas sus características, Complu$d o c$ es una de las principales fuentes españolas de información científica por su amplia cobertura (con más de 1.000 revistas analizadas de las más diversas áreas del conocimiento y 160.000 registros), gratuidad, facilidad de consulta, rápida actualización, servicio de DSI gratuito vía correo electrónico, edición automática de boletines de sumarios electrónicos y posibilidad de envío reprográfico de los artículos mediante préstamo interbibliotecario sujeto al sistema de tarifas de Rebiun (Red de Bibliotecas Universitarias).

\section{«Compludoc es una de las prin- cipales fuentes españolas de in- formación científica por su am- plia cobertura, gratuidad, facili- dad de consulta, rápida actuali- zación, etc.»}

Dentro del panorama analizado Compludoc sería comparable por sus objetivos, cobertura temática y tipología documental con otras bases de sumarios orientadas a la alerta informativa (como las ofrecidas a sus clientes por los distribuidores de publicaciones periódicas o los servicios de obtención de documentos). Sin embargo, no se han encontrado ejemplos gratuitos de similar magnitud generados desde una biblioteca con recursos propios.

En cuanto a su cobertura geográfica, las más prestigiosas bases de datos generales sobre revistas científicas españolas son las ofrecidas por el Cindoc (Centro de Información y Documentación Científica), del Csic, pero, hoy por hoy, su política de distribución está sometida a tarifación, siendo difícil la comparación en el contexto de este artículo. Por otro lado, sus metas son más ambiciosas (al incorporar un análisis documental detallado que incluye descriptores y resúmenes de un gran número de las referencias) y su cobertura es general (intenta cubrir todas las publicaciones científicas españolas) frente a la local de Compludoc (las principales revistas recibidas en la Universidad Complutense). Como contrapartida, la amplitud de sus objetivos ocasiona ciertos retrasos que justifican su coexistencia con una base de sumarios de la naturaleza de Complu$d o c$, dado que permite una disposición más rápida de las referencias tal y como denota el análisis realizado 
en abril de 2000 y que puede observarse en las tablas de la figura 3.

Aunque estos datos son difícilmente comparables, sí son orientativos y denotan una mayor prontitud de Compludoc a la hora de ofrecer las referencias. A nuestro entender, ambos recursos se complementan por su diferente grado de cobertura, tipología documental y objetivos.

Por otro lado, algunas empresas e instituciones bibliotecarias han expresado su interés por la adquisición de los datos de Compludoc (caso del Cbuc Consorcio de Bibliotecas Universitarias de Cataluña- que incluye parte de sus registros en su Base de dades de sumaris). Lo lógico sería una planificación cooperativa que evitara duplicar esfuerzos permitiendo un incremento del número de revistas españolas o extranjeras disponibles gratuitamente para los investigadores. Es deseable, por su similitud documental y gratuidad de acceso, algún tipo de concierto junto a la Base de dades de sumaris del Cbuc y la realizada por la Universidad Politécnica de Cataluña para ofrecer un completo servicio de sumarios de las principales revistas científicas suscritas en España.

\section{«Se ha barajado la posibilidad de ampliar el proyecto con la in- clusión de las publicaciones ex- tranjeras de mayor impacto de forma gratuita, la puesta en fun- cionamiento de tesauros inte- ractivos y la inserción de enla- ces al texto completo de los ar- tículos de aquellas con versión electrónica suscrita»}

Finalmente, sus carencias son:

- La falta de datos respecto a ciertas áreas cuyos centros no han podido incorporarse aún al proyecto, aunque lo hacen paulatinamente (Educación, por ejemplo, se acaba de incorporar),

- la limitación temática derivada del hecho de que la $U C M$ no abarca dentro de sus estudios a las carreras técnicas, y

- la falta de homogeneidad y profundidad del análisis documental que depende de los recursos o prioridades de cada centro participante.

5. Expectativas. Se ha ampliado el campo de cobertura a todas las áreas científicas de la UCM: ciencias sociales, humanidades, ciencias de la salud y ciencias. De igual modo, algunos de los centros empiezan a realizar un control bibliográfico más exhaustivo (es el caso de económicas o biblioteconomía y documentación, que incluyen un resumen del contenido de los artículos). Otros centros (Económicas) trabajan en la creación de bases de datos retrospectivas que recojan lo publicado en las revistas españolas de carácter científico cuyos fondos se encuentren en la $U C M$ sin límite temporal. También se ha barajado la posibilidad de ampliar el proyecto con la inclusión de las publicaciones extranjeras de mayor impacto de forma gratuita, la puesta en funcionamiento de tesauros interactivos (ya activo para el área de economía) y la inserción de enlaces al texto completo de los artículos de aquellas con versión electrónica suscrita.

6. A modo de corolario. Hasta aquí, una pequeña revisión del panorama del acceso gratuito a la información bibliográfica. Pese a encontrarnos en un marco joven, las perspectivas son inmejorables pues la tendencia muestra un incremento de la gratuidad en servicios cada vez más interactivos que girarán sobre el acceso no a las referencias, sino a los contenidos. En este ámbito, el papel de las bibliotecas al servicio del público no puede situarse por detrás del mundo comercial, sino que deben garantizar la accesibilidad sin restricciones. Este entorno parece propio de un estudio más detallado, del que aquí sólo podemos señalar algunas de sus singularidades: portales de información generados desde bibliotecas que permitirán el acceso gratuito a servicios y colecciones digitales propias, junto a las que se ofrecerán recopilaciones de las ofertadas sin restricciones por otras instituciones o empresas, a fin de garantizar su difusión y conocimiento mediante servicios personalizados por los propios usuarios. Lógicamente, ello únicamente es posible desde posiciones donde los consorcios no sean sólo meras asociaciones para ahorrar dinero, sino el ámbito que exteriorice el apoyo gubernamental a la difusión de la información.

\section{Bibliografía}

Bosseau, Don L.; Martin, Susan K.; Hirshon, Arnold. "Libraries, consortia, and change management". En: Journal of Academic Librarianship, 1999, v. 25, n. 2, pp. 124-126.

Coffman, Steve. "Building earth's largest library: driving into the future". En: Searcher, 1999, v. 7, n. 3, pp. 34-45.

Coffman, Steve. "What if you ran your library like a bookstore?". En: American libraries, 1998, v. 29, n. 3, pp. 40-44.

Jacsó, Peter. "Fee or free? The next level of competition". En: Information today, 1999, noviembre, v. 16, n. 10, pp. 34-35.

Jacsó, Peter. "What is digital librarianship?". En: Computers in libraries, 2000, v. 20, n. 1 , pp. 54-55.

Tomaiulo, Nicholas G. "Free bibliographic information on the web". En: Searcher, 1999, abril, v. 7, n. 4, pp. 20-25.

José Antonio Magán Wals. Director de la biblioteca de la Facultad de ciencias económicas y empresariales. Universidad Complutense de Madrid.

magan@buc.ucm.es 